\title{
Reconstruction of Time-Of-Flight PET Data with the STIR reconstruction framework
}

\author{
Nikos Efthimiou, Member, IEEE, Elise Emond, Student Member, IEEE, Chris Cawthorne, \\ Charalampos Tsoumpas, Senior Member, IEEE and Kris Thielemans, Senior Member, IEEE
}

\begin{abstract}
This manuscript gives an update on the integration of capabilities to handle Time-Of-Flight (TOF) Positron Emission Tomography (PET) data into the STIR image reconstruction toolkit. Most infrastructure classes related to raw PET data have been extended, utilities to handle listmode or sinogram data can now handle TOF information, and TOF modelling has been added to the ray-tracing system matrix. This extension required many modifications in the low level code base of STIR, making it non-trivial and error-prone. Therefore, a thorough validation was required. In this work we provide a description of the modifications to the software and the testing. In addition, initial results of the correctness of the extension are reported. Using Monte Carlo simulations, as well as cylindrical and XCAT phantoms analytically projected, we calculate the contrast recovery ratio over a wide range of iterations. The results demonstrate the benefits of TOF under different configurations, which are in good agreement with literature.
\end{abstract}

\section{INTRODUCTION}

$\mathbf{S}$ TIR is an open source and freely distributed image reconstruction framework [1] supporting Positron Emission Tomograhy (PET) and Single Photon Emission Tomography (SPECT) data. It is widely used by many research groups around the world. STIR provides analytical and iterative reconstruction algorithms for both projection and listmode data, motion correction, scatter estimation etc. However, it currently does not support Time-of-Flight (TOF) PET data.

TOF PET estimates the path length difference between two annihilation $\gamma$ photons of a same pair by measuring the detection time difference. This information gives us an estimation of the most likely position of the annihilation along a projection line [2].

The purpose of this manuscript is to give a comprehensive description of the TOF implementation in the STIR framework. This required non-trivial modifications to the software

Manuscript received November 10, 2017. This project was supported by the European Cooperation for Science and Technology Action TD1401: Fast Advanced Scintillation Timing (http://cern.ch/fast-cost), GlaxoSmithKline R\&D (BIDS3000030921), the Daisy Appeal Charity and CCP PET-MR (EPSRC grant EP/M022587/1). The first two authors contributed equally to this work.

Nikos Efthimiou is with the School of Life Sciences, Faculty of Health Sciences, University of Hull, Hull HU6 7RX, UK (e-mail: n.efthymiou@hull.ac.uk).

Elise Emond is with the Institute of Nuclear Medicine, University College London, London NW12BU, UK (e-mail: elise.emond.16@ucl.ac.uk).

Chris Cawthorne is with the School of Life Sciences, Faculty of Health Sciences, University of Hull, Hull HU6 7RX, UK (e-mail: c.cawthorne@hull.ac.uk).

Charalampos Tsoumpas is with the Division of Biomedical Imaging, University of Leeds, Leeds LS2 9JT, UK (e-mail: c.tsoumpas@leeds.ac.uk).

Kris Thielemans is with the Institute of Nuclear Medicine, University College London, London NW1 2BU, UK (e-mail: k.thielemans@ucl.ac.uk). so a careful validation was needed. We have previously presented initial results of the application of the timing information on the LOR applied to simulated listmode data using the Siemens mCT geometry [3]. This work covered only listmode reconstructions. In the version described here, support of projection data (TOF sinograms) is added. This addition to STIR is useful both for validation of the TOF capabilities in STIR and of course to be able to reconstruct non-listmode data from clinical scanners.

\section{Materials And Methods}

\section{A. TOF Detection model}

Let $p_{i t ; j}$ be the probability that an annihilation photon pair emitted in voxel $v_{j}$ is detected by the detector pair $i$ represented by $\mathrm{DET}_{\mathrm{A}}$ and $\mathrm{DET}_{\mathrm{B}}$ in Figure 1 in TOF bin $t$. The detected time difference between the two photon arrivals determines the location $k$ along the LOR. To find $p_{i t ; j}$ we need to integrate the detection probability along the line over the line segment $k_{t}, k_{t+1}$ corresponding to the TOF bin. We assume that the TOF detection probability can be considered to be constant over the size of the voxel. The centre of each voxel in the LOR is projected onto the line connecting the two detectors and $p_{i t ; j}$ is calculated according to Equation 1 using the error function erf, assuming that the uncertainty on the timing is Gaussian. $\operatorname{proj}_{v j}$ is the projection of voxel $v_{j}$ on the line connecting the two detectors and determines the width of the timing kernel.

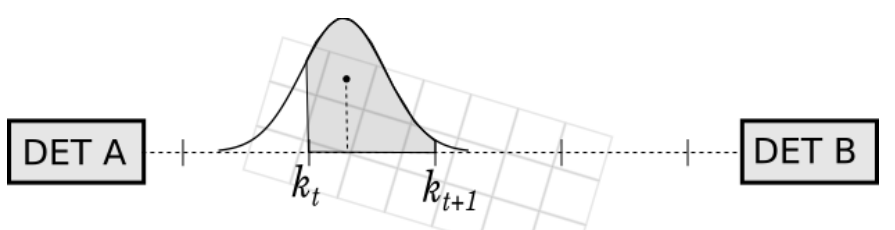

Fig. 1: Illustration of the TOF kernel application

$p_{i t ; j}=\left(\operatorname{erf}\left(\frac{k_{t+1}-\operatorname{proj}_{v j}}{\sigma \sqrt{2}}\right)-\operatorname{erf}\left(\frac{k_{t}-\operatorname{proj}_{v j}}{\sigma \sqrt{2}}\right)\right) / 2 \times p_{i j}^{\text {nonTOF }}$

This formula ensures that summing over the TOF bins, the nonTOF probability will be recovered.

\section{B. Implementation}

1) Data structures: The Scanner templates were extended with three new variables: max_num_of_timing_bins, which 
holds the number of TOF bins without any TOF mashing, size_timing_bin, which holds the size in picoseconds of the unmashed TOF bin and timing_resolution, the FWHM (in ps) of the timing response. Furthermore, the classes related to projection data, such as ProjDataInfo and ProjData were extended to provide support for the TOF dimension. A TOF projection data array has one more dimension. In order to introduce the smallest possible amount of changes, the ProjData members were extended such as get_sinogram to have an extra argument corresponding to the timing position index, such that they return a data-structure of the same dimension as in the non-TOF case. Interfile IO was extended, allowing specifying the order of the coordinates within the header file.

List-mode classes were adapted to handle data from clinical scanners with TOF information. An implementation for the GE Signa PET/MR is provided, but data formats for other scanners will be added. In addition, the ROOT file format used by the Monte Carlo simulator GATE [4] is now supported. Unlisting of TOF listmode data to TOF sinograms is also implemented (lm_to_projdata).

2) Classes for acquisition modelling and reconstruction:

The ForwardProjectorByBin and BackProjectorByBin classes were extended to take into account TOF information. Currently, only the projectors based on a system response matrix (SRM), i.e. using a class in the ProjMatrixByBin hierarchy, are TOF-ready.

The most commonly used SRM model in STIR is ProjMatrixByBinUsingRayTracing, which is based on a variation of Siddons algorithm [5], optionally with multiple rays per detector pair. However, as opposed to implementing TOF support for a specific type of SRM, in the current implementation, the TOF kernel is applied on top of the nonTOF probabilities as in eq. 1 in ProjMatrixByBin. This has the advantage that the TOF modification can be used for any SRM matrix in STIR. In normal operation, the nonTOF probabilities are precalculated, stored in a cache to avoid computational overhead. Particular care was taken to be able to cope with different timing resolution, width of the TOF bins and "TOF mashing", where adjacent TOF bins are added together to reduce data size and calculation time.

Due to the modular structure of STIR, all iterative reconstruction algorithms in STIR can now handle TOF data without modification to the actual reconstruction code.

3) Supporting utilities: A set of provided utilities had to be upgraded to include loops over the TOF bins, examples include list_projdata_info, which allows the user to extract some basic sinogram statistics, poisson_noise to add Poisson noise to the data, stir_math to perform basic operations on images and sinograms (non-TOF and TOF) etc.

\section{Validation}

To validate the software, we used both analytical simulations (forward-projecting an image, including attenuation, using STIR) and Monte Carlo simulations, using the GATE toolkit [4]. We show results using the scanner characteristics from the GE Discovery PET/CT 690 as an example but other scanners have been implemented as well. This scanner has a TOF resolution of $550 \mathrm{ps}, 55 \mathrm{TOF}$ bins of width $89 \mathrm{ps}$. Three other time resolutions were also simulated for illustration: $300 \mathrm{ps}$, $400 \mathrm{ps}$ and 500ps. TOF mashing was applied to reduce the number of TOF bins to 11 in some of the simulations.

For the analytical simulations shown here, Poisson noise was added, at a level comparable to a real acquisition.

\section{RESUltS}

In order to validate the previously stated erf model, eq. 1, subset sensitivity images (backprojection of $1 \mathrm{~s}$ ) were calculated using on the one hand the non-TOF backprojector and on the other hand several TOF backprojectors (varying timing resolutions of $300,400,500$ and 550 ps, with 11 TOF bins, i.e. TOF mashing factor 5). They were then compared and the results showed that the mean difference was $0.0012 \pm 0.0007 \%$, which is negligible. Also, as an additional check we summed the forward projected data over the TOF dimension to form a non-TOF sinogram. The difference between the newly-created non-TOF sinogram and the original non-TOF sinogram is also negligible (less than $0.001 \%$ for all timing resolutions used).

To further validate the TOF projection matrix, GATE was used to simulate 12 point sources in different locations. The scanner TOF characteristics were modified to simulate a close to perfect time resolution (time resolution: $75 \mathrm{ps}, 275$ TOF bins of width $17.8 \mathrm{ps}$ ). We compared the centre of mass of maximum probability backprojections of the ROOT data with the location of the point sources, as shown for two of the point source simulations on the Figure 2. In all cases, the centre of mass was placed within the image resolution range of the original position.

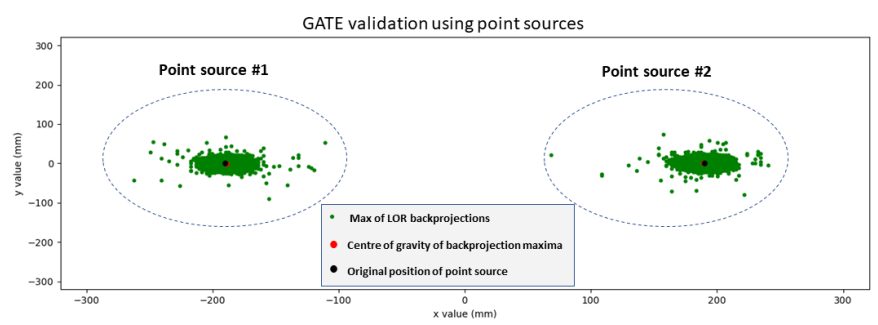

Fig. 2: Centre of mass of maximum probability backprojections of the ROOT data with the location of the point sources.

Forward projection to non-TOF and TOF sinograms (with 5 TOF bins) of an emission image consisting of an oblique plane, located near the centre of the FOV (L40xW15xH0.8 $\mathrm{cm}^{3}$ ), are shown in Figure 3.

An image of a cylindrical phantom was forward projected and attenuated. The sinograms were then reconstructed with OSEM (18 subsets, up to 108 iterations). In Figure 4, reconstructed images at the 18th sub-iteration are illustrated. TOF reconstruction provides sharper images with higher noise levels. Without TOF the border between the inner and outer phantoms are blurred and the contrast is lower.

Reconstructed XCAT [6] images of the thorax with TOF, at the 72th sub-iteration, are sharper than their non-TOF counterparts, with better defined edges. When an additional Gaussian 


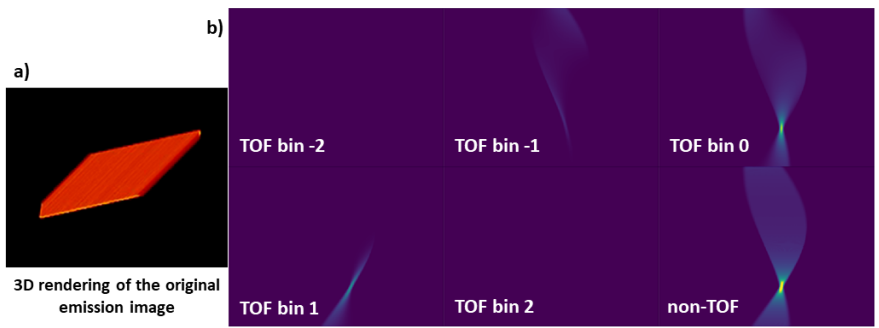

Fig. 3: TOF sinograms of an oblique plane.

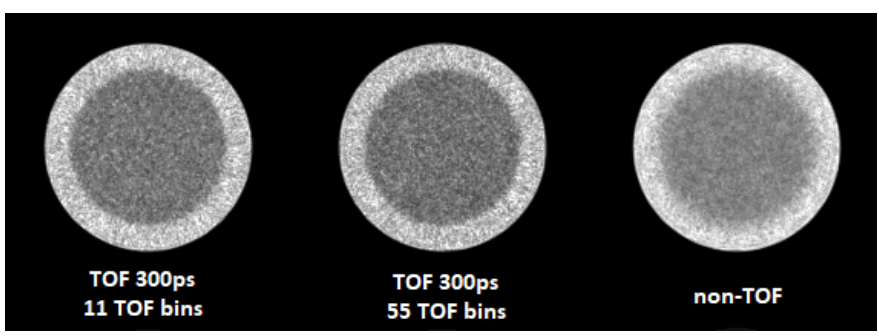

Fig. 4: Analytically simulated cylindrical phantom, reconstructed with different TOF configurations and non-TOF.

3D postfilter with $6.4 \mathrm{~mm}$ FWHM is used, TOF reconstruction presents better noise properties with preservation of the edge boundaries (as shown on Figure 5). We used 2 ROIs: on the lesion located in the left lung and the whole right lung, obtaining the Coefficient of Variation $(\mathrm{CoV})$ in the right lung, calculated as (standard deviation in the right lung)/(mean value in the right lung), and the Signal-to-Noise Ratio (SNR) as (lesion mean activity value)/(right lung standard deviation).

Lesion mean value over CoV shows that TOF reconstructions converges faster and at lower noise levels than non-TOF (Figure 6(a)). When, postfiltering is applied the convergence slows down and the noise is reduced. In the presented case studies, in terms of CRC, TOF and non-TOF do not demonstrate significant difference, when the images are postfiltered.

SNR decreases at each sub-iteration, as images get noisier. In the presented case studies, TOF does not show significant benefits. With postfiltering the SNR slightly increases between the 18th and the 36th sub-iterations, as the filtering slows down the convergence step, and then slowly decreases.

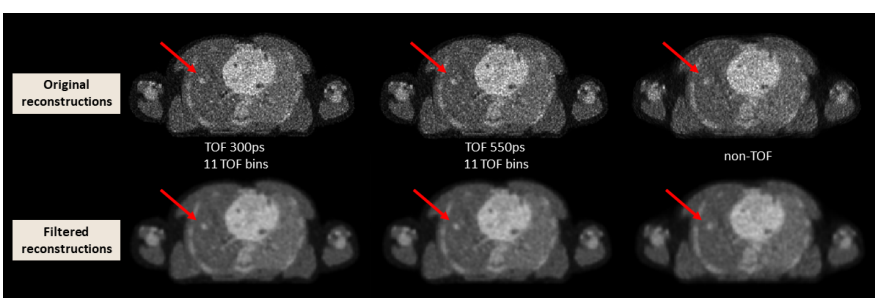

Fig. 5: Anthropomorphic XCAT phantom reconstructed for without TOF and under two TOF configurations. At the bottom row an additional Gaussian postfilter was applied.

Limited FOV in the case of modern PET/MR scanner, or scan of large patients may compromise the image quality of the reconstructed images, as shadowing and wrong quantification can be introduced. As demonstrated in Figure 7, when smaller

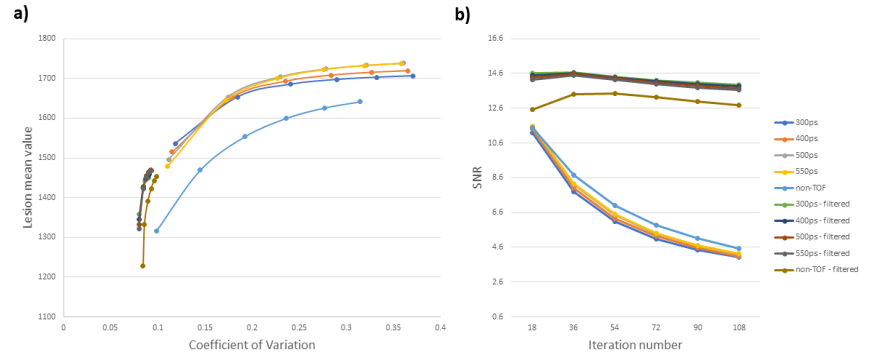

Fig. 6: a) Lesion mean activity vs CoV b) SNR vs sub-iteration number

FOV size is used, TOF images are somewhat less affected by the truncation, as expected. This indicates that STIR handles the truncation appropriately.

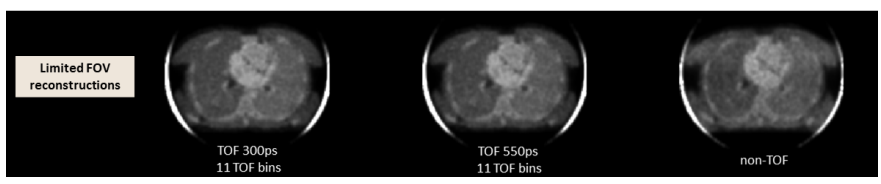

Fig. 7: Reconstruction of truncated images due to limited FOV.

\section{CONCLusion}

STIR is an open source and freely distributed image reconstruction framework. It is widely used by many research groups around the word. It provides analytical and iterative reconstruction algorithms, for projection data and listmode, motion correction, scatter estimation etc. The new extension to TOF data resides at a level low enough to affect most of the provided functionalities in the framework. Therefore, the upgrade needed careful validation.

In this work we presented validation of the TOF projection comparing with GATE Monte Carlo data. In addition, initial results for TOF projection data reconstruction are described. Initial results from two phantoms were forward projected, using the Discovery PET/CT 690 scanner specifications, attenuated and reconstructed. STIR performed as expected. Reconstruction with TOF speeds up the convergence. As the same model is used to process the TOF information we have checked that the reconstruction of projection and listmode data will produce the exact same results.

This extension to TOF will be made available as part of STIR in the near future. TOF scatter modeling remains for future work.

\section{REFERENCES}

[1] K. Thielemans, "STIR: Software for Tomographic Image Reconstruction Release 2," Phys. Med. Biol., vol. 57, no. 4, pp. 867-883, 2012.

[2] T. Tomitani, "Image-reconstruction and noise evaluation in photon timeof-flight assisted positron emission tomography," IEEE Trans. Nucl. Sci., vol. 28 , no. 9.

[3] N. Efthimiou, "Initial Validation of Time-Of-Flight List-Mode MLEM and OSEM Reconstruction Algorithms in STIR framework, using Monte Carlo simulated data," IEEE MIC, 2016.

[4] S. Jan, "GATE: A simulation toolkit for PET and SPECT," Phys. Med. Biol., vol. 49, no. 19.

[5] L. R. Siddon, "Fast calculation of the exact radiological path for a threedimensional CT array," Med. Phys., vol. 12, no. 2, pp. 252-255, 1985. 
[6] W. Segars, "4D XCAT phantom for multimodality imaging research." Med. Phys., vol. 37, no. 9. 